\title{
Dynamic contrast enhanced computed tomography: a precise technique for identifying and localising pancreatic necrosis
}

\author{
Michael Larvin, Alan G Chalmers, Mìchael J McMahon
}

University Department of Surgery and Department of Diagnostic Radiology, General Infirmary, Leeds LS1 3EX

Michael Larvin, FRCs, research fellow

Alan G Chalmers, FRCR, consultant radiologist

Michael J McMahon, FRCS, consultant surgeon

Correspondence to: Mr McMahon.

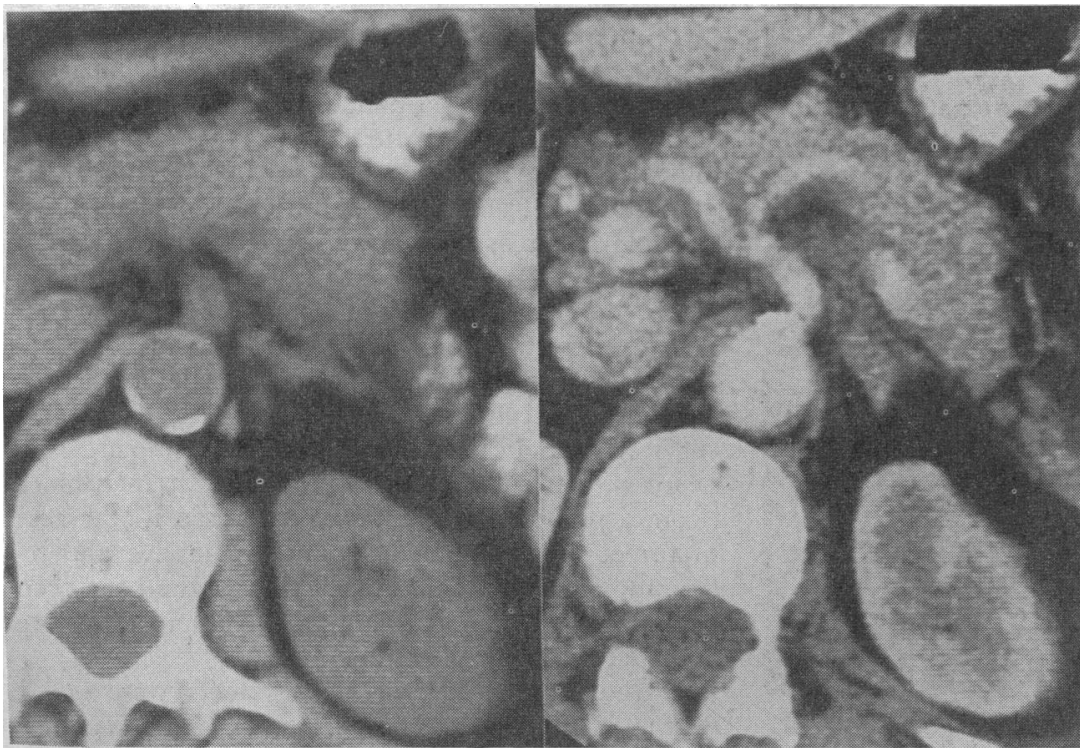

FIG 1-Matched computed tomograms before (left) and after (right) administration of intravenous contrast, showing increased density of normal pancreas and other perfused structures

Results-Dynamic computed tomography correctly localised pancreatic necrosis in 11 patients (confirmed at laparotomy in nine and at necropsy in two). Of nine patients with low enhancement of peripancreatic tissues alone, eight recovered after conservative management; necropsy confirmed viable pancreas and necrosis of peripancreatic fat in one patient. Of 40 patients with normal contrast enhancement, none required laparotomy to debride pancreatic necrosis.

Conclusion-Dynamic contrast enhanced computed tomography seems to be a safe and accurate method of identifying and localising pancreatic and

\begin{abstract}
Objective-To evaluate dynamic contrast enhanced computed tomography for detecting and localising pancreatic necrosis in acute pancreatitis.

Design-Prospective evaluation with blind reporting of scans.

Setting-Single teaching hospital.

Patients-60 Consecutive patients with acute pancreatitis suspected to have pancreatic necrosis because of major organ system failure (13); slow recovery five to seven days after admission with raised scores on the acute physiological and chronic health evaluation (APACHE-II) system (27); or findings on previous ultrasonography or computed tomography (20).

Main outcome measure-Pancreatic necrosis proved histologically - that is, $>\mathbf{3 0} \mathrm{g}$ necrotic tissue debrided at laparotomy (for life threatening sepsis or peritonitis) or necropsy.
\end{abstract}

peripancreatic necrosis, which cannot be predicted accurately by conventional imaging techniques.

\section{Introduction}

Fatal acute pancreatitis is often associated with septic complications of pancreatic necrosis, ${ }^{12}$ which we define as a collection of solid necrotic tissue ${ }^{3}$ rather than pus (pancreatic abscess) or pancreatic juice (pseudocyst). Collections of pus and pancreatic juice are associated with lower morbidity and mortality. ${ }^{45}$ Survival of patients with pancreatic necrosis often depends on surgical debridement of necrotic tissue. ${ }^{2-46}$ Before surgery the presence and extent of pancreatic necrosis must be identified. This cannot be achieved by clinical assessment, ${ }^{7}$ laboratory criteria, ${ }^{7.9}$ or ultrasonography, ${ }^{810}$ and even at laparotomy surgeons cannot differentiate accurately between necrotic and viable tissue $^{11}$ or between necrotic pancreas and necrotic peripancreatic fat. ${ }^{12}$

Computed tomography shows characteristic changes in pancreatic and peripancreatic tissues during acute pancreatitis, ${ }^{1314}$ and although a correlation with outcome has been shown, ${ }^{1315} 16$ the extent to which the results of computed tomography are prognostic is disputed. ${ }^{17-19}$ The pancreas is richly vascularised and its density in computed tomograms is considerably increased, or enhanced, after patients have been given intravenous contrast medium (fig 1). The enhancement is measured by calculating the increase in density in defined areas. Kivisaari et al evaluated contrast enhanced computed tomography in 29 patients who had pancreatectomy for fulminant pancreatitis. ${ }^{20}$ Enhancement was less than 30 Hounsfield units in 21 patients with pancreatic necrosis but exceeded 40 Hounsfield units in eight patients with oedematous pancreatitis. Slow scanning times (10 seconds) and a single scanning level, however, made accurate localisation impossible. Multiple level scanning has also been evaluated ${ }^{8}$ : five of 62 patients with low enhancement did not have pancreatic necrosis, and 10 of 15 patients with normal enhancement had substantial necrosis. Scanning times of 5-10 seconds may have precluded precise evaluation.

Modern computed tomographic scanners, which have improved resolution, shorter scanning times, and dynamic scanning modes, allow sequential scans to be obtained rapidly. Non-ionic contrast media have minimised renal and cardiovascular complications. We examined a technique that incorporated these advances and report on its performance in identifying and localising pancreatic necrosis in acute pancreatitis.

\section{Patients and methods}

All the patients had been admitted to hospital with a clinical diagnosis of acute pancreatitis and had plasma 
amylase activities $>1200 \mathrm{IU} / \mathrm{l}$ (Phadebas, Pharmacia, Milton Keynes). Patients had dynamic computed tomography if pancreatic necrosis was suspected because of major organ system failure (box); slow recovery five to seven days after admission, with scores on the acute physiological and chronic health evaluation system (APACHE-II) $\geqslant 8^{22}$; or evidence of severe pancreatitis on ultrasonography or tomography taken before referral suggesting severe pancreatitis. Predictions of pancreatic necrosis based on dynamic computed tomograms were confirmed if histological examination of tissue obtained by laparotomy or necropsy showed more than $30 \mathrm{~g}$ necrotic tissue. ${ }^{3}$ Indications for surgery were based not on the results of computed tomography but on increasing sepsis or signs of peritonitis. Patients who died had necropsies, and survivors were followed up for 15 to 43 months (median 30 months).

We used a CT-9800 scanner (IGE Medical Systems, Slough) with a dynamic facility, which permitted detailed imaging of pancreatic and peripancreatic tissues during the brief period of enhancement after bolus injection of contrast medium..$^{20}$ Before scanning, $500-750 \mathrm{ml}$ of $3 \%$ Gastrografin solution $(10 \%$ sodium diatrizoate, $66 \%$ meglumine diatrizoate; Schering Pharmaceuticals, Burgess Hill) was given to each patient either orally or through a nasogastric tube. Scans of $10 \mathrm{~mm}$ thickness were obtained every $20 \mathrm{~mm}$ from the domes of the diaphragm caudally. Scanning levels for contrast enhanced computed tomography were selected, and a rapid sequence of 10-14 contiguous or alternate $5 \mathrm{~mm}$ sections was obtained immediately after intravenous injection of $100-150 \mathrm{ml}$ of $76 \%$ iopamidol (Niopam 370, E Merck, Alton; equivalent to a dosage of $500-750 \mathrm{mg}$ iodine $/ \mathrm{kg}$ ) over 30 to 75 seconds. Patients were scanned at end expiration for two seconds with a six second delay between scans to permit respiration. After reconstruction contrast enhancement was assessed throughout the pancreas and peripancreatic tissues. As in previous studies enhancement of less than 30 Hounsfield units was considered abnormal, enhancement exceeding 40 Hounsfield units was considered normal, and enhancement of 30-40 Hounsfield units was considered equivocal..$^{20}$ The results of tomography were analysed immediately by a single consultant radiologist, who commented on enhancement of peripancreatic tissues and the pancreatic head, body, and tail.

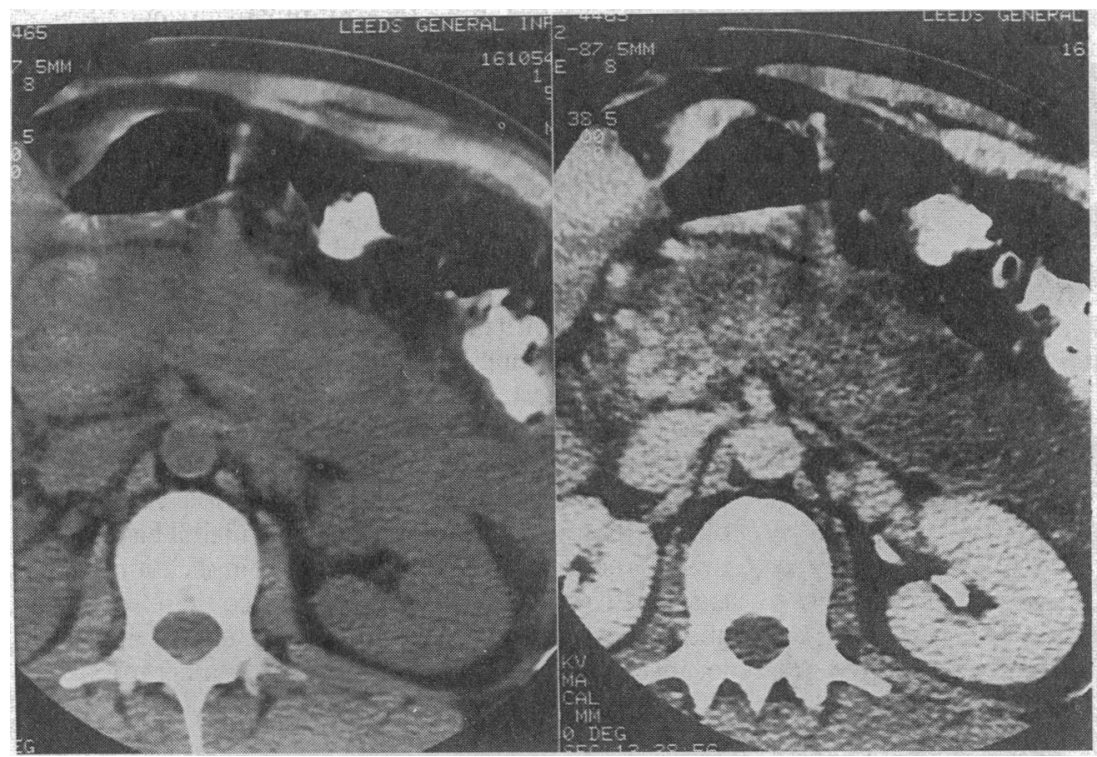

FIG 2-Matched computed tomograms of patient who subsequently had debridement of necrotic body and tail of pancreas. Tomogram obtained before contrast was administered (left) shows diffuse pancreatic swelling and peripancreatic inflammation, whereas that obtained afterwards (right) shows minimal enhancement of body and no enhancement of the tail, both of which were necrotic

\section{Definitions of major organ system failure}

Arterial hypoxia-Arterial oxygen pressure $<8$ $\mathrm{kPa}$ necessitating $>24$ hours' oxygen treatment or ventilation

Renal failure-Mean urine volume $<20 \mathrm{ml} / \mathrm{h}$ for $24 \mathrm{~h}$

Refractory hypotension-Systolic blood pressure $<100 \mathrm{~mm} \mathrm{Hg}$ despite administration of pressor agents

Coagulopathy-Prothrombin time, activated partial thromboplastin time, or thrombin time exceeding twice control values; or platelet count $<20 \times 10^{9} / 1$; or fibrinogen concentration $<1 \mathrm{~g} / \mathrm{l}$; or concentration of fibrinogen degradation products exceeding twice normal value

\section{Results}

Sixty patients fulfilled our selection criteria during April 1986 to June 1988: 13 had major organ system failure, 27 had a slow recovery with raised APACHE-II scores, and 20 had had severe disease on previous imaging. During the study 109 other patients were admitted to the hospital with acute pancreatitis but did not have computed tomography. None of them required laparotomy for pancreatic necrosis.

Computed tomography was performed between one hour and seven days (median three days) after admission to hospital. Eleven patients had areas of abnormal pancreatic enhancement. Pancreatic necrosis was confirmed either at laparotomy (nine patients) or at necropsy (two). Of the 11 patients, six had necrosis restricted to the pancreas (fig 2) and five had pancreatic and peripancreatic necrosis. In all cases the anatomical distribution of necrosis precisely matched that indicated by dynamic computed tomography (table).

Relation between radiological evidence of pancreatic enhancement and findings at laparotomy or necropsy. Values are numbers of patients

\begin{tabular}{lccc}
\hline & $\begin{array}{c}\text { Patients with contrast } \\
\text { enhancement }<30 \\
\text { Hounsfield units }\end{array}$ & \multicolumn{2}{c}{$\begin{array}{c}\text { Pancreatic necrosis } \\
\text { confirmed by: }\end{array}$} \\
\cline { 3 - 4 } Area of pancreas & 6 & Laparotomy & Necropsy \\
\hline Head, body, or tail & 1 & 5 & 1 \\
Head and body & 1 & 1 & \\
Body and tail & 3 & 1 & 1 \\
Head, body, and tail & & 2 &
\end{tabular}

A further nine patients had normal pancreatic enhancement but abnormal peripancreatic enhancement (fig 3 (top)): eight recovered after conservative management and peripancreatic necrosis could not be confirmed; one patient died and peripancreatic necropsy confirmed a viable pancreas with necrosis of peripancreatic fat (fig 3 (bottom)).

Of the remaining 40 patients, 36 had normal enhancement throughout and four had equivocal pancreatic enhancement initially which had returned to normal on rescanning 48 hours later. All 40 patients survived without requiring debridement of pancreatic necrosis, but collections of fluid were drained in 12 (from a pseudocyst in nine and a pancreatic abscess in three; by percutaneous puncture in five and by operation in seven). All 12 collections of fluid had been noted on computed tomography obtained before administration of contrast medium.

No adverse cardiac, or renal effects were noted during 134 dynamic computed tomographic examinations in the 60 patients. One patient vomited while receiving the intravenous contrast medium, and angina 


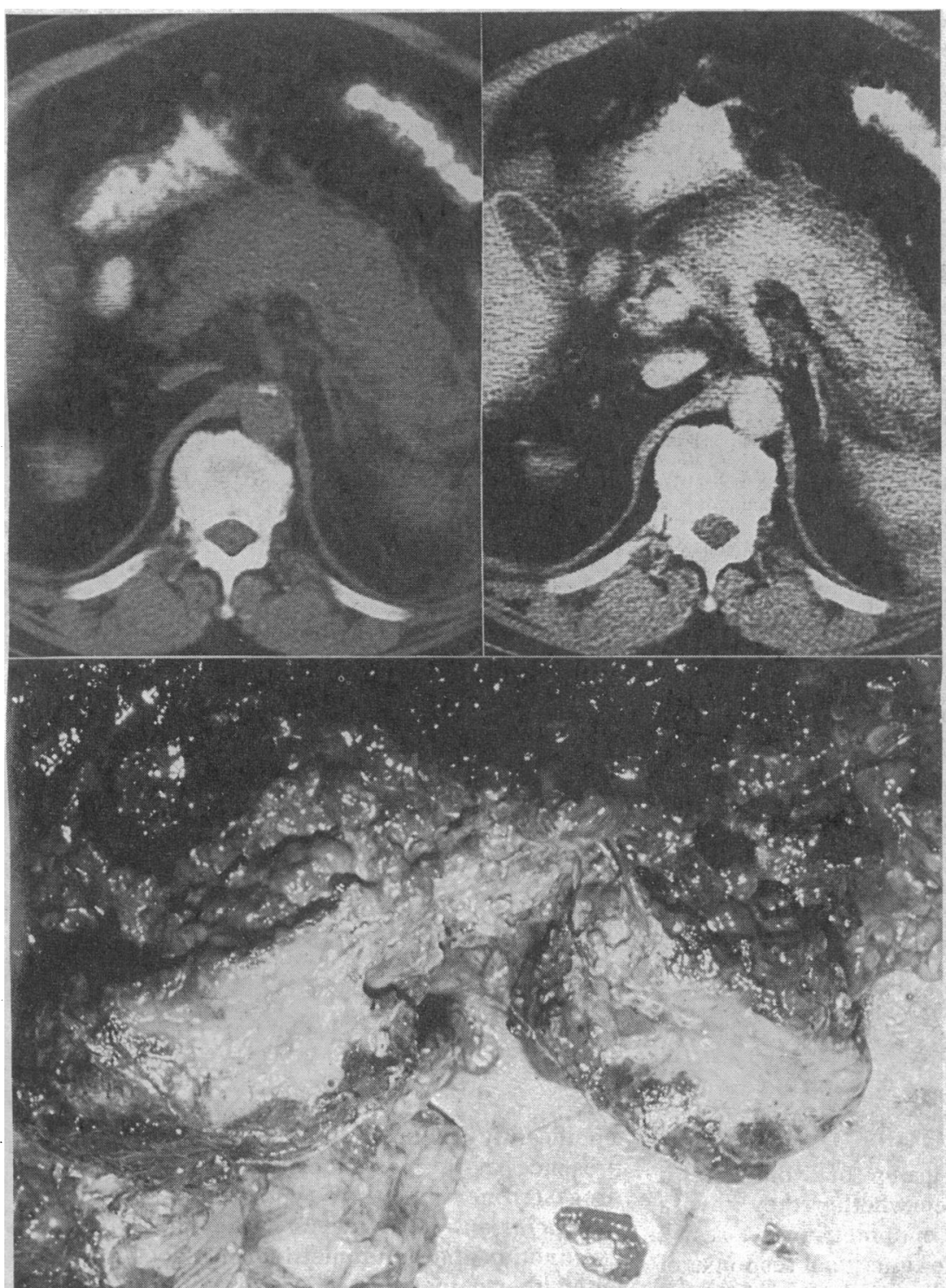

FIG 3-(Top) Matched computed tomograms obtained before (left) and after (right) administration of contrast. Tomogram obtained after contrast was given shows rind of poorly enhancing tissue surrounding pancreas anteriorly and inferiorly, which is not visible in scan obtained before contrast was given. (Bottom) Necropsy specimen from same patient, oriented anatomically and sectioned vertically through midbody of pancreas. Histological examination confirmed pancreatic viability, but rim of darker tissue, which corresponded to area of low enhancement, consisted of necrotic fat

was precipitated in another patient; in neither case were there serious sequelae. Several patients had watery diarrhoea after scanning.

\section{Discussion}

We evaluated a technique of dynamic contrast enhanced computed tomography for detecting pancreatic necrosis. Patients had to be given contrast medium orally to delineate the pancreas and peripancreatic tissues from the adjacent bowel. The diarrhoea experienced by some patients probably related to the high osmolality of this contrast medium as, more recently, it has been prevented by giving patients a non-ionic medium (3\% iohexol; diluted Omnipaque 350 , Nycomed, Birmingham). We used a non-ionic intravenous contrast medium throughout because many patients had cardiovascular instability or renal failure; both are contraindications to giving ionic media. The high dose of intravenous contrast medium and rapid injection were essential to sustain enhancement for one to two minutes, the time taken to image the entire pancreatic area with a modern computed tomographic scanner with a dynamic facility.

Three patterns of abnormal enhancement emerged: abnormal pancreatic enhancement probably rep- resents necrosis of devitalised pancreas (central necrosis); abnormal pancreatic and peripancreatic enhancement represents extension of necrosis beyond the gland (extended central necrosis); and abnormal enhancement of peripancreatic tissue alone represents necrosis of fat and connective tissue due to exudation of activated pancreatic enzymes (peripheral necrosis). Central and extended central necrosis was confirmed histologically. Peripheral necrosis sometimes appeared as a "rind" around the pancreas and sometimes as a crenated or scalloped pattern, suggesting necrosis of fat in the periphery of the gland. As the decision to perform laparotomy was based on clinical progress we could confirm the pattern of peripheral necrosis only in the patient who died.

Patients with abnormal or equivocal contrast enhancement had dynamic computed tomography at least weekly until surgical or radiological intervention was undertaken or recovery occurred. The unaided recovery of four patients with equivocal enhancement suggests that areas of poorly perfused or ischaemic pancreas may improve spontaneously provided that perfusion does not become critically low. No such improvement was noted in patients with pancreatic enhancement of less than 30 Hounsfield units, in all of whom pancreatic necrosis was debrided. Although abnormal enhancement was often obvious from inspection of tomograms obtained before and after the administration of contrast medium, our findings emphasise the need to quantify enhancement accurately.

Pancreatic enhancement after intravenous administration of contrast medium may be influenced by the nature and volume of the contrast medium, injection speed, cardiac output, blood volume, splanchnic perfusion, and elimination of the medium from the circulation. Capillary blood flow was the principal determinant of enhancement in pigs with acute pancreatitis, ${ }^{24}$ and microangiography of human pancreatectomy specimens confirmed that microcirculatory damage in pancreatic necrosis corresponds with abnormal enhancement. ${ }^{25}$

A clearer understanding of the pathological evolution of pancreatic necrosis is now emerging, ${ }^{124525}$ and the survival of patients treated in specialist units has improved, ${ }^{2-46}$ possibly because patients likely to require surgical debridement are more accurately identified..$^{2-4813}$ Pancreatic necrosis may be sterile or infected, ${ }^{1-526}$ and whereas the patients with infected necrosis are typically the most severely ill,' sterile pancreatic necrosis can be lethal. ${ }^{-3}$ Computed tomography cannot differentiate between infected and sterile necrosis, ${ }^{5}$ but percutaneous aspiration guided by computed tomography can do so reliably. ${ }^{26}$

Dynamic contrast enhanced computed tomography identified and localised pancreatic necrosis precisely and proved safe when patients were given a non-ionic intravenous contrast medium. Doubts about safety will probably persist, but we believe that dynamic computed tomography should be used when pancreatic necrosis is suspected even in severely ill patients because of the precise information it provides. It did not indicate when an operation was necessary, but when surgery was indicated clinically it provided information that facilitated a less invasive surgical procedure and ensured that all necrotic tissue was located and debrided adequately. ${ }^{3}$

We thank the consultant surgeons at this hospital for allowing us to investigate their patients and the consultan clinicians of other hospitals (Airedale General Hospital, Bradford Royal Infirmary, Doncaster Royal Infirmary, Jersey General Hospital, Huddersfield Royal Infirmary, Royal Halifax Infirmary, Staincliffe General Hospital, Wharfedale General Hospital, York District Hospital) who referred 
patients who participated in this study. This project was supported by Bayer UK.

1 Beger HG, Bittner R, Block S, Buchler M. Bacterial contamination of pancreatic necrosis A prospective clinical study. Gastroenterology 1986;91: $433-8$

2 Frey CF, Bradley EL, Beger HG. Progress in acute pancreatitis. Surg Gynecol Obstet 1988;167:282-6.

3 Larvin M, Chalmers AG, Robinson PJ, McMahon MJ. Debridement and closed cavity irrigation for the treatment of pancreatic necrosis. $\mathrm{Br} f \mathrm{Sur}$ 1989;76:465-71

4 Bradley EL. Management of infected pancreatic necrosis by open drainage Ann Surg 1987;206:542-50.

5 Bittner R, Block S, Buchler M, Beger HG. Pancreatic abscess and infected pancreatic necrosis. Different local septic complications in acute pancreatitis. Dig Dis Sci 1987;32:1082-7.

6 Beger HG, Krautzberger W, Bittner R, Block S, Buchler M. Results of urgical treatment of necrotising pancreatitis. World F Surg 1985;9:972-9.

Corfield AP, Cooper MJ, Williamson RCN, et al. Prediction of severity in acute pancreatitis. Prospective comparison of three prognostic indices. acute pancreatitis.
Lancet $1985 ; \mathrm{ii}: 403-7$

8 Block S, Maier W, Bittner R, Buchler M, Malfertheiner P, Beger HG Identification of pancreas necrosis in severe acute pancreatitis: imaging procedures versus clinical staging. Gut 1986;27:1035-42.

9 Leese T, Shaw D, Holliday M. Prognostic markers in acute pancreatitis: can pancreatic necrosis be predicted? Ann R Coll Surg Engl 1988;70:227-32.

10 Swobodnick $W$. Controversies and limitations of ultrasonography in acute pancreatitis. In: Malfertheiner $\mathrm{P}$, Ditschuneit $\mathrm{H}$, eds. Diagnostic procedures in pancreatic disease. Berlin: Springer Verlag, 1985:32-6.

11 Nordback I, Pessi T, Auvinen O, Autio V. Determination of necrosis in necrotising pancreatitis. Br f Surg 1985;72:225-7.

12 Howard JM, Wagner SM. Pancreatography after recovery from massive pancreatic necrosis. Ann Surg 1989;209:31-5.

13 Ranson JHC, Balthazar E, Caccavale R, Cooper M. Computed tomography and the prediction of pancreatic abscess in acute pancreatitis. Ann Surg 1985;201:656-65.
4 Lawson TL. Acute pancreatitis and its complications. Computed tomography and sonography. Radiol Clin North Am 1983;21:495-513.

15 Schroder T, Kivisaari L, Somer K, Standertskjold-Nordenstam C-G, Kivilaakso $\mathrm{E}$, Lempinen $\mathrm{M}$. Significance of extrapancreatic findings in computed tomography of acute pancreatitis. Eur 7 Radiol 1985;5:273-5.

16 Nordestegaard AG, Wilson SE, Williams RA. Early computerised tomography as a predictor of outcome in acute pancreatitis. Am $\mathcal{J}$ Surg 1986;152:127-32.

17 Rotmann N, Bonnet F, Larde D, Fagniez P'-L. Computerised tomography in the evaluation of late complications of acute pancreatitis. Am $\mathcal{F}$ Surg 1986; 152:286-9.

18 Vernacchia FS, Jeffrey RB, Federle MP, et al. Pancreatic abscess: the predictive value of early CT. Radiology 1987;162:435-8

19 Lọndon NJ, Neoptolemos JP, Lavelle J, Bailey I, James D. Contrast enhanced computed tomography scanning and prediction of severity of acute pancreatitis: a prospective study. Brf Surg 1989;76:268-72.

20 Kivisaari L, Somer K, Standertskjold-Nordenstam C-G, Kivilaakso E, Lempinen $M$. A new method for the diagnosis of acute haemorrhagic necrotising pancreatitis using contrast enhanced CT scanning. Gastrointest Radiol 1984;9:27-30.

21 Knaus WA, Draper EA, Wagner DP, Zimmerman JE. APACHE-II: a severity of disease classification. Crit Care Med 1985:13:818-29.

22 Larvin M, McMahon MJ. APACHE-II score for assessment and monitoring of acute pancreatitis. Lancet 1989;ii:201-5.

23 Schroder T, Kivisaari L, Somer K, et al. The clinical significance of contrast enhanced computed tomography in acute pancreatitis. Ann Chir Gynaecol 1984;73:268-72.

24 Nuutinen P. Contrast enhanced computed tomography in acute oedematous pancreatitis. Surgical Research Communications 1987;1:251-9.

25 Nuutinen P, Kivisaari L, Schroder T. Contrast enhanced computed tomography and microangiography of the pancreas in acute human hemorrhagic necrotising pancreatitis. Pancreas 1988; 3:53-60.

26 Gerzof SG, Banks PA, Robbins AH, et al. Early diagnosis of pancreatic infection by computed tomography guided aspiration. Gastroenterology $1987 ; 93: 1315-20$.

(Accepted 22 February 1990)
Department of

Biomedical Science,

University of Sheffield,

Sheffield S10 2TN

R A Graham, BSC, research assistant

The late A W Rogers, DSC, formerly professor

P Dockery, PHD, lecturer

Departments of Obstetrics and Gynaecology,

Biochemistry, and

Molecular Biology,

University of Manchester,

St Mary's Hospital,

Manchester M13 0JH

$M$ W Seif, PHD, clinical tutor

J D Aplin, PHD, lecturer

Department of Obstetrics and Gynaecology, University of Sheffield, Jessop Hospital for Women, Sheffield S3 7RE T C Li, PHD, clinical lecturer I D Cooke, FRCOG, professor

Correspondence to:

Dr J D Aplin, Department of Obstetrics and

Gynaecology, Research

Floor, St Mary's Hospital,

Manchester M13 0JH.

Br.Med F 1990;300:1428-31

\title{
An endometrial factor in unexplained infertility
}

\author{
R A Graham, M W Seif, J D Aplin, T C Li, I D Cooke, A W Rogers, P Dockery
}

\section{Abstract}

Objective-To study a group of women with unexplained infertility to see whether they have a defect that is intrinsic to the endometrium.

Design-Evaluation of the functional response of the endometrium by examining endometrial biopsy specimens using immunohistochemical methods in a group of women with unexplained infertility and in a control group of women with normal fertility.

Patients -27 Women with unexplained infertility (average age 33.2); median duration of infertility five years. A control group of $\mathbf{4 4}$ women with normal fertility (average age 33.8 ) who were requesting sterilisation or reversal of sterilisation.

Setting-Infertility clinic, Jessop Hospital for Women, Sheffield.

Intervention-Secretory phase endometrial biopsy specimens were taken, with informed consent, as an outpatient procedure.

Main outcome measures -Immunohistochemistry with monoclonal antibody D9B1, was used to assess the production and secretion of an oligosaccharide epitope produced by endometrial gland cells between two and seven days after the luteinising hormone surge. A reflected light measuring system was used to assess the amount of epitope within the gland cells, and in the gland lumen.

Results - In the control group of women, mean reflected light measurements at the cell base and cell apex peaked at three and five days after the luteinising hormone surge respectively, and in the gland lumen the epitope accumulated rapidly from three days, reaching a peak at seven days. In the women with infertility the peaks of epitope at the cell base and cell apex were lower, broader, and delayed in onset, and the build up of epitope in the gland lumen was retarded. The synthesis and secretion of the epitope in the women with infertility was therefore significantly reduced and delayed, even in the presence of normal concentrations of circulating progesterone.

Conclusions-The results suggest that a primary dysfunction of the endometrium might be associated with hitherto unexplained infertility.

\section{Introduction}

Substantial numbers of patients with unexplained infertility attend infertility clinics, with reported prevalences ranging from $6 \%$ to almost $60 \%$. $^{1}$ The hormonal profiles of women with unexplained infertility have been much studied and are often normal, but in contrast, the endometrium has been comparatively unexplored as a factor in infertility. This has been justified by the widespread belief that endometrial morphology provides no more than a simple and faithful reflection of the ovarian cycle. The use of the endometrial biopsy specimen as an index of ovulation has been largely superseded by non-invasive, quantitative, sensitive, endocrine assays, and failure of the endometrium to respond fully to a normal ovarian stimulus has remained hypothetical.

The secretory differentiation of the endometrial epithelium has been characterised by raising monoclonal antibodies to hormonally regulated cell surface and secretory components. ${ }^{34}$ The monoclonal antibody D9B1 binds to a peptide associated sialooligosaccharide of the sulphated lactosaminoglycan family, ${ }^{56}$ which is secreted by gland cells mainly between three and seven days after the luteinising hormone surge. ${ }^{4}$ Study of the defective luteal phase has shown that there is a strong correlation between infertility and reduced production of the D9B1 epitope ${ }^{7}$ this deficiency may occur against a background of normal secretory differentiation as assessed by conventional histology. On the basis of these and 